\title{
Sommes d'exponentielles et principe de l'hyperbole
}

\author{
par \\ Louis Goubin (Orsay)
}

1. Introduction. Beaucoup de problèmes d'arithmétique sont liés à des questions d'indépendance entre les structures multiplicative et additive des entiers. L'étude du comportement asymptotique des sommes d'exponentielles de la forme

$$
\sum_{n \leq x} f(n) e(n \theta)
$$

où $f$ est une fonction multiplicative et $e(n \theta):=\exp (2 i \pi n \theta)$ est additive, permet d'obtenir des renseignements intéressants sur cette «indépendance».

Il est alors naturel de se demander pour quel type de fonction multiplicative $f$ on a, pour tout irrationnel $\theta$,

$$
\sum_{n \leq x} f(n) e(n \theta)=o\left(\sum_{n \leq x} f(n)\right) .
$$

On notera $\mathcal{D}$ l'ensemble de ces fonctions et $\mathcal{M}$ l'ensemble des fonctions multiplicatives.

Dans le cas des fonctions multiplicatives à valeurs dans le disque unité, on dispose du résultat suivant, dû à H. Daboussi (cf. [3], [4] ou [10], p. 392) :

ThÉORÈme A (Daboussi). Pour tout $\theta$ irrationnel on a, uniformément pour $f$ multiplicative à valeurs dans le disque unité,

$$
\sum_{n \leq x} f(n) e(n \theta)=o(x) .
$$

Or on sait d'après un théorème de $\mathrm{H}$. Delange ([5]) que si $f$ est multiplicative à valeurs dans le disque unité, elle admet une valeur moyenne non nulle si et seulement si les deux conditions suivantes sont vérifiées :

$$
\sum_{p} \frac{1-f(p)}{p} \text { converge } \quad \text { et } \quad \exists k \geq 1, \quad f\left(2^{k}\right) \neq-1 .
$$


On obtient ainsi une première classe de fonctions qui vérifient (2) :

$$
\left\{f \in \mathcal{M}:|f| \leq 1, \sum_{p} \frac{1-f(p)}{p} \text { converge, } \exists k \geq 1, f\left(2^{k}\right) \neq-1\right\} \subset \mathcal{D} \text {. }
$$

Plus généralement, K. H. Indlekofer ([8]) a obtenu (3) en supposant seulement que $f$ est uniformément sommable.

DÉfinition. Une fonction arithmétique $f$ est dite uniformément sommable si on a

$$
\lim _{y \rightarrow \infty}\left(\sup _{x \geq 1} \frac{1}{x} \sum_{\substack{n \leq x \\|f(n)| \geq y}}|f(n)|\right)=0 .
$$

On notera $\mathcal{L}^{*}$ l'ensemble des fonctions multiplicatives uniformément sommables.

Indlekofer a montré ([8]) que les fonctions de $\mathcal{L}^{*}$ qui ont une valeur moyenne non nulle sont caractérisées par les propriétés suivantes :

- $\sum_{p}(f(p)-1) / p$ converge,

- $\quad \sum_{p}|f(p)-1|^{2} / p<\infty$ $|f(p)| \leq 3 / 2$

- $\quad \sum_{p}|f(p)| / p<\infty$ $|f(p)-1| \geq 1 / 2$

- $\sum_{p} \sum_{k \geq 2}\left|f\left(p^{k}\right)\right| / p^{k}<\infty$,

- $\forall p, 1+\sum_{k=1}^{\infty} f\left(p^{k}\right) / p^{k} \neq 0$.

On obtient ainsi une nouvelle classe de fonctions contenue dans $\mathcal{D}$.

En utilisant le principe de l'hyperbole de Dirichlet, Chowla ([1]) a montré que la fonction $d$ (nombre de diviseurs) est aussi dans $\mathcal{D}$ :

ThÉorÈme B (Chowla). Pour tout $\theta$ irrationnel, on a

$$
\sum_{n \leq x} d(n) e(n \theta)=o(x \log x) .
$$

En fait, Erdős ([7]) a montré qu'en général, on peut obtenir une majoration bien meilleure :

ThÉORÈme C (Erdős). Pour presque tout $\theta$, on a

$$
\sum_{n \leq x} d(n) e(n \theta)=\mathcal{O}(\sqrt{x} \log x) .
$$


Par ailleurs Y. Dupain, R. R. Hall et G. Tenenbaum ([6]) ont montré que les fonctions $y^{\Omega}$ sont dans la classe $\mathcal{D}$ lorsque $0<y<2$ (ici $\Omega(n)$ désigne le nombre de facteurs premiers de $n$ comptés avec multiplicité) :

ThÉorème D (Dupain, Hall, Tenenbaum). Si $0<y<2$, pour tout $\theta$ irrationnel, on a

$$
\sum_{n \leq x} y^{\Omega(n)} e(n \theta)=o\left(\sum_{n \leq x} y^{\Omega(n)}\right) .
$$

Leur démonstration - de nature analytique - s'appuie entre autres sur une estimation du type «Siegel-Walfisz» du comportement de $y^{\Omega}$ dans les progressions arithmétiques de raison «petite».

Dans ce qui précède, les fonctions multiplicatives considérées sont à valeurs réelles positives. Pour des fonctions $f$ plus générales, seuls des résultats du type (3) semblent accessibles.

Dans cette direction, H. Daboussi (cf. [2]) a généralisé (5) de la façon suivante :

THÉORÈME E (Daboussi). Soit $f$ une fonction complètement multiplicative telle que pour tout nombre premier $p$ on ait $|f(p)|=y$, avec $0<y<2$. Alors pour tout $\theta$ irrationnel on a

$$
\sum_{n \leq x} f(n) e(n \theta)=o\left(\sum_{n \leq x}|f(n)|\right) .
$$

2. Résultats. Le premier objectif de cet article est d'obtenir un résultat de stabilité de la classe $\mathcal{D}$ par convolution. On montrera le résultat général suivant :

THÉORÈME 1. Soient $f$ et $h$ deux fonctions multiplicatives telles que

(H1) $\sum_{p}(h(p)-1) / p, \quad \sum_{\substack{p \\|h(p)| \leq 3 / 2}}|h(p)-1|^{2} / p$,

$$
\sum_{\substack{p \\|h(p)-1| \geq 1 / 2}}|h(p)| / p \text { et } \sum_{p} \sum_{k \geq 2}\left|h\left(p^{k}\right)\right| / p^{k} \text { convergent }
$$

(H2) $\forall p, 1+\sum_{k=1}^{\infty} h\left(p^{k}\right) / p^{k} \neq 0$,

(F1) $f \geq 0$,

(F2) $\sum_{n \leq x} f(n)=o\left(x \sum_{n \leq x} f(n) / n\right)$,

(F3) $f \in \mathcal{D}$.

Alors, si on pose $g:=f * h$, on a $g \in \mathcal{D}$. 
Dans la pratique, on utilisera souvent des hypothèses plus faibles :

Corollaire 1. Soient $f$ et $h$ deux fonctions multiplicatives telles que

$\left(\mathrm{H}^{\prime} 1\right)|h| \leq 1$ et $\sum_{p}(1-h(p)) / p$ converge,

$\left(\mathrm{H}^{\prime} 2\right) \exists k \geq 1, h\left(2^{k}\right) \neq-1$,

(F1) $f \geq 0$,

(F2) $\sum_{n \leq x} f(n)=o\left(x \sum_{n \leq x} f(n) / n\right)$,

(F3) $f \in \mathcal{D}$.

Alors, si on pose $g:=f * h$, on a $g \in \mathcal{D}$.

En particulier, $h=1$ convient, ce qui donne :

Corollaire 2. Soit $f$ une fonction multiplicative telle que :

(F1) $f \geq 0$

(F2) $\sum_{n \leq x} f(n)=o\left(x \sum_{n \leq x} f(n) / n\right)$.

On suppose que $f \in \mathcal{D}$. Alors, si on pose $g:=f * 1$, on a $g \in \mathcal{D}$.

On sait (voir par exemple [10], p. 340) que la condition (F2) est vérifiée dès qu'il existe des constantes $\lambda_{1}>0$ et $0 \leq \lambda_{2}<2$ telles que

$$
\forall p \text { premier, } \forall k \geq 1, \quad 0 \leq f\left(p^{k}\right) \leq \lambda_{1} \lambda_{2}^{k-1} \text {. }
$$

Considérons les fonctions diviseurs généralisées $d_{l}\left(l \in \mathbb{N}^{*}\right)$, où $d_{l}(n)$ est le nombre de manières de décomposer $n$ en produit de $l$ entiers non nuls. En remarquant que

$$
\sum_{n \leq x} d_{l}(n) \sim x(\log x)^{l-1}
$$

et

$$
x \sum_{n \leq x} \frac{d_{l}(n)}{n} \sim x(\log x)^{l},
$$

on constate que $f=d_{l}$ entre dans le champ d'application du corollaire 2. On voit donc que si $d_{l}$ est dans $\mathcal{D}$, alors $d_{l+1}$ aussi. Comme $d_{1}$ est évidemment dans $\mathcal{D}$, on en déduit :

Corollaire 3. Pour tout $l \in \mathbb{N}^{*}$ et tout $\theta$ irrationnel, on a

$$
\sum_{n \leq x} d_{l}(n) e(n \theta)=o\left(\sum_{n \leq x} d_{l}(n)\right)=o\left(x(\log x)^{l-1}\right) .
$$

Remarquons que cette assertion peut également se déduire des résultats de [6]. En effet, la méthode employée par Dupain, Hall et Tenenbaum dans 
cet article pour établir (5) fonctionne sans changement (cf. la remarque page 406) dans le cas de $\omega(n)$ et fournit

$$
\sum_{n \leq x} y^{\omega(n)} e(n \theta)=o\left(x(\log x)^{y-1}\right) \quad(\theta \in \mathbb{R} \backslash \mathbb{Q}, y>0) .
$$

Si on considère la fonction $d_{y}$, définie par

$$
\sum_{n=1}^{\infty} \frac{d_{y}(n)}{n^{s}}=\zeta(s)^{y} \quad(\operatorname{Re} s>1)
$$

on peut écrire $d_{y}=y^{\omega} * h$, avec

$$
\sum_{m=1}^{\infty} \frac{|h(m)|}{m}<\infty
$$

ce qui permet de conclure que $d_{y}$ est dans $\mathcal{D}$ pour tout $y>0$.

L'intérêt est ici d'avoir obtenu le corollaire 3 par des moyens élémentaires, sans faire appel à des estimations du type «Siegel-Walfisz».

En combinant le théorème $\mathrm{E}$ - dont la démonstration utilise (5) - et le principe mis en évidence de stabilité de la classe $\mathcal{D}$, on montrera ensuite le résultat suivant :

THÉORÈme 2. Soit $y>0$. On suppose que $f$, multiplicative, vérifie les conditions

(i) $|f(p)| \leq y$ pour tout nombre premier $p$,

(ii) la série $\sum_{p} \sum_{\nu=2}^{\infty}\left(\left|f\left(p^{\nu}\right)\right| / p^{\nu}\right)\left(\log p^{\nu}\right)^{\max (1-y, 0)}$ converge.

Alors pour tout $\theta$ irrationnel, on a

$$
\sum_{n \leq x} f(n) e(n \theta)=o\left(x(\log x)^{y-1}\right) .
$$

On voit facilement que ce résultat contient les théorèmes $\mathrm{A}, \mathrm{B}, \mathrm{D}, \mathrm{E}$ et le corollaire 3 , ainsi que le fait que $y^{\omega}$ et $d_{y}$ sont dans $\mathcal{D}$ pour $y>0$.

Dans le cas où $0<y<2$, le théorème 2 découle assez facilement $\mathrm{du}$ théorème $\mathrm{E}$, par un argument de convolution. Un examen attentif de la démonstration dans [2] montre que la preuve du théorème $\mathrm{E}$ - en son état actuel — n'est transposable à la situation d'une fonction multiplicative générale satisfaisant les conditions (i) et (ii) que lorsque $0<y<2$. En effet, le résultat provient, en dernier ressort, du fait que la quantité

$$
\prod_{p \leq T}\left(1-\frac{1}{p}\right)^{2 y}\left(1+\frac{y^{2}}{p}\right)
$$

tend vers 0 quand $T$ tend vers $\infty$, ce qui n'est vrai que pour $y<2$. 
C'est pourquoi dans le cas général, on emploiera une méthode analogue à celle de la démonstration du théorème 1 , reposant sur la stabilité par convolution du comportement des sommes d'exponentielles mises en jeu.

Le théorème 2 ne donne un renseignement non trivial que si

$$
\sum_{p} \frac{y-|f(p)|}{p}<\infty .
$$

Sous cette hypothèse supplémentaire, le résultat peut s'écrire de la façon suivante :

Corollaire 4. Soit $y>0$. On suppose que $f$, multiplicative, vérifie les conditions

(i) $|f(p)| \leq y$ pour tout nombre premier $p$,

(ii) la série $\sum_{p} \sum_{\nu=2}^{\infty}\left(\left|f\left(p^{\nu}\right)\right| / p^{\nu}\right)\left(\log p^{\nu}\right)^{\max (1-y, 0)}$ converge,

(iii) la série $\sum_{p}(y-|f(p)|) / p$ converge.

Alors pour tout $\theta$ irrationnel, on a

$$
\sum_{n \leq x} f(n) e(n \theta)=o\left(\sum_{n \leq x}|f(n)|\right) .
$$

Par ailleurs, on ne peut pas supprimer la condition

$$
\sum_{p} \sum_{\nu=2}^{\infty} \frac{\left|f\left(p^{\nu}\right)\right|}{p^{\nu}}\left(\log p^{\nu}\right)^{\max (1-y, 0)}<\infty
$$

du corollaire 4. Ainsi, on montrera que la fonction multiplicative $2^{\Omega}-$ qui vérifie les hypothèses (i) et (iii) pour $y=2$ - n'appartient pas à la classe $\mathcal{D}$ :

ThÉORÈme 3. Il existe un irrationnel $\theta$ tel que l'on ait, quand $x$ tend vers $\infty$,

$$
\sum_{n \leq x} 2^{\Omega(n)} e(n \theta)=\Omega\left(\sum_{n \leq x} 2^{\Omega(n)}\right) .
$$

De même on peut montrer que pour $y \geq 2, y^{\Omega}$ n'est pas dans $\mathcal{D}$. On constate donc que la restriction $0<y<2$ dans le théorème $\mathrm{D}$ est inévitable.

Remerciements. Je tiens à remercier le referee anonyme pour des remarques qui ont permis de simplifier certains points - notamment la démonstration du théorème 2 dans le cas $0<y<2$ - et de clarifier la démonstration du théorème 1 .

3. Démonstration du théorème 1 . Pour tout réel $\theta$, et tout $x \geq 1$, on note

$$
G(x, \theta):=\sum_{n \leq x} g(n) e(n \theta) .
$$


On pose $G(x):=G(x, 0)$, dont le comportement peut être relié à celui de $f$ de la manière suivante :

LEMme 1. Sous les hypothèses du théorème 1 , on a

$$
G(x) \asymp x \sum_{m \leq x} \frac{f(m)}{m} .
$$

Preuve. D'après Indlekofer ([8]), les hypothèses (H1) et (H2) montrent que $h$ est absolument sommable et vérifie, pour un certain $\lambda \in \mathbb{C}^{*}$,

$$
\sum_{n \leq x} h(n) \sim \lambda x
$$

Ensuite, en écrivant

$$
\lambda x \sum_{m \leq x} \frac{f(m)}{m}-G(x)=\sum_{m \leq x} f(m)\left[\frac{\lambda x}{m}-\sum_{l \leq x / m} h(l)\right],
$$

puis en séparant en deux la somme, selon la position de $m$ par rapport à $x / K$, on obtient

$$
\begin{aligned}
& \lambda x \sum_{m \leq x} \frac{f(m)}{m}-G(x) \\
& \leq \sum_{m \leq x / K} f(m)\left|\frac{\lambda x}{m}-\sum_{l \leq x / m} h(l)\right|+\left(K|\lambda|+\sum_{l \leq K}|h(l)|\right) \sum_{m \leq x} f(m) .
\end{aligned}
$$

Un $\varepsilon$ strictement positif étant donné, on peut d'après (7) choisir $K=K(\varepsilon)$ pour que la valeur absolue dans le premier terme soit majorée par $\varepsilon x /(2 m)$. A cause de l'hypothèse (F2), le second terme de (8) est alors majoré par $(\varepsilon x / 2) \sum_{m \leq x} f(m) / m$ pour $x \geq x_{0}(\varepsilon)$, ce qui fournit le résultat attendu.

(a) C a s o ù $\sum_{m=1}^{\infty} f(m) / m<\infty$. En écrivant la somme d'exponentielles sous la forme

$$
G(x, \theta)=\sum_{m \leq x} f(m) \sum_{l \leq x / m} h(l)
$$

et en séparant en deux termes suivant la position de $m$ par rapport à $K$, on obtient

(9) $|G(x, \theta)| \leq \sum_{m \leq K} f(m)\left|\sum_{l \leq x / m} h(l) e(l m \theta)\right|+\sum_{K<m \leq x} f(m)\left[\sum_{l \leq x / m}|h(l)|\right]$.

Du fait que $h$ est uniformément sommable, on déduit facilement que le terme entre crochets et majoré - à une constante près - par $x / m$ (cf. [8]).

Si on fixe $\varepsilon>0$, l'hypothèse supplémentaire faite ici sur $f$ permet de majorer le second terme de (9) par $\varepsilon x / 2$, à condition de choisir $K=K(\varepsilon)$ assez grand. 
Pour le premier terme, on a vu que $h$ - étant uniformément sommable et de valeur moyenne non nulle - appartient à la classe $\mathcal{D}$. Tous les $m \theta$ étant irrationnels, on en déduit que l'on peut majorer le premier terme par $\varepsilon x / 2$ pour $x \geq x_{0}(\varepsilon)$.

On obtient ainsi $G(x, \theta)=o(G(x))$ car le lemme 1, joint à l'hypothèse faite sur $f$, donne $G(x) \asymp x$.

(b) Ca s o ù $\sum_{m=1}^{\infty} f(m) / m=\infty$. D'après Indlekofer ([8]), le fait que $h$ soit uniformément sommable et de valeur moyenne non nulle permet d'en déduire une écriture plus pratique sous la forme d'une convolution :

LEMME 2. Il existe des fonctions multiplicatives $\widetilde{h}$ et $\psi$ et une constante $A>0$ telles que $h=\widetilde{h} * \psi$, avec $\sum_{n}|\psi(n)| / n<\infty$ et

$$
\sum_{n \leq x}|\widetilde{h}(n)|^{2} \leq A^{2} x, \quad \forall p,|\widetilde{h}(p)| \leq A .
$$

On notera, de manière analogue à $G(x, \theta)$

$$
\widetilde{H}(x, \theta):=\sum_{n \leq x} \widetilde{h}(n) e(n \theta) .
$$

Remarquons que l'inégalité de Cauchy-Schwarz donne immédiatement $\widetilde{H}(x, 0) \ll x$.

Les propriétés de $\widetilde{h}$ permettent d'obtenir des renseignements intéressants sur son comportement dans les sommes d'exponentielles (cf. [9]) :

ThÉORÈme F (Montgomery, Vaughan). Si $|\theta-a / q| \leq 1 / q^{2},(a, q)=1$ et $2 \leq B \leq q \leq x / B$, alors

$$
\widetilde{H}(x, \theta) \ll \frac{x}{\log x}+x B^{-1 / 2}(\log B)^{3 / 2} .
$$

En utilisant le lemme 2, on peut écrire

$$
G(x, \theta)=\sum_{l m \leq x} \psi(l) f(m) \widetilde{H}\left(\frac{x}{l m}, \operatorname{lm} \theta\right) .
$$

D'après le lemme 1 , il suffit de montrer que pour tout $\varepsilon>0$ et pour $x \geq x_{0}(\varepsilon)$, le membre de droite de (11) est

$$
\ll \varepsilon x \sum_{m \leq x} \frac{f(m)}{m} .
$$

La convergence de la série $\sum_{l}|\psi(l)| / l$ et la majoration $\widetilde{H}(x, 0) \ll x$ permettent de supposer $l \leq L$, où $L=L(\varepsilon)$ est choisi assez grand. L'hypothèse

$$
\sum_{m \leq x} f(m)=o\left(x \sum_{m \leq x} \frac{f(m)}{m}\right)
$$


permet également de restreindre la sommation de (11) au domaine $l m \leq$ $x / x_{1}(\varepsilon)$ pour toute constante fixée $x_{1}(\varepsilon)$. Il reste donc à montrer que pour $y>x_{2}(\varepsilon)$, on a

$$
\sum_{m \leq y / x_{1}(\varepsilon)} f(m) \widetilde{H}\left(\frac{y}{m}, m \theta\right) \ll \varepsilon y \sum_{m \leq y} \frac{f(m)}{m} .
$$

Or le résultat de Montgomery et Vaughan implique la propriété suivante :

Pour chaque $\varepsilon>0$, on a $\widetilde{H}(x, \theta) \ll \varepsilon x$ dès que $x>x_{1}(\varepsilon)$ et

$$
\{\theta\} \notin E(\varepsilon):=\bigcup_{q \leq B} \bigcup_{\substack{1 \leq a<q \\(a, q)=1}}\left[\frac{a}{q}-\frac{1}{q Q} ; \frac{a}{q}+\frac{1}{q Q}\right],
$$

avec $B=1 / \varepsilon^{3}$ et $Q=\left\lfloor 1 / \varepsilon^{4}\right\rfloor$. On peut prendre par exemple $x_{1}(\varepsilon)=$ $\exp (1 / \varepsilon)$.

On voit bien que la sous-somme de (12) correspondant à $\{m \theta\} \notin E(\varepsilon)$ est majorée par le membre de droite. Il suffit donc d'évaluer, pour $y>x_{2}(\varepsilon)$,

$$
\sum_{\substack{m \leq y \\\{\theta m\} \in E(\varepsilon)}} \frac{f(m)}{m} .
$$

Dans ce but, on majore la fonction indicatrice de $E(\varepsilon)$ par le polynôme trigonométrique

$$
\begin{aligned}
C \sum_{q \leq B} \sum_{\substack{1 \leq a<q \\
(a, q)=1}}\left(\frac{\sin \pi \theta q Q}{q Q \sin (\pi(\theta-a / q))}\right)^{2} & \\
= & \frac{C}{Q} \sum_{q \leq B} \frac{1}{q} \sum_{\substack{1 \leq a<q \\
(a, q)=1}} \sum_{|k| \leq q Q}\left(1-\frac{|k|}{q Q}\right) \cos \left(2 \pi k\left(\theta-\frac{a}{q}\right)\right),
\end{aligned}
$$

où $C$ est une constante absolue. On reporte dans (13), et on intervertit les sommations. Comme $f$ est dans $\mathcal{D}$, on voit — par sommation d'Abel — que la contribution de chaque $k \neq 0$ est $o\left(\sum_{m \leq y} f(m) / m\right)$. On obtient donc que (13) est $\ll(\varepsilon+o(1)) \sum_{m \leq y} f(m) / m$, ce qui fournit bien le résultat attendu.

4. Démonstration du corollaire 1. Il suffit de montrer que les hypothèses (H1) et (H2) de la proposition 2 sont vérifiées.

D'après $\left(\mathrm{H}^{\prime} 2\right), \exists k \geq 1, \operatorname{Re}\left(h\left(2^{k}\right)\right)>-1$. Donc

$$
\operatorname{Re} \sum_{k=1}^{\infty} \frac{h\left(2^{k}\right)}{2^{k}}>\sum_{k=1}^{\infty} \frac{-1}{2^{k}}=-1 .
$$


De plus,

$$
\forall p \geq 3, \quad \operatorname{Re} \sum_{k=1}^{\infty} \frac{h\left(p^{k}\right)}{p^{k}} \geq \sum_{k=1}^{\infty} \frac{-1}{p^{k}}=\frac{-1}{p-1}>-1,
$$

ce qui donne (H2).

Pour prouver (H1), on utilise l'inégalité classique :

$$
\forall u \in \mathbb{C}, \quad|u| \leq 1 \Rightarrow|u-1|^{2} \leq 2 \operatorname{Re}(1-u) .
$$

En particulier,

$$
\forall p, \quad|h(p)-1|^{2} \leq 2(1-\operatorname{Re} h(p)) .
$$

Or par hypothèse $\sum_{p}(1-\operatorname{Re} h(p)) / p<\infty$. Donc

$$
\sum_{p} \frac{|h(p)-1|^{2}}{p}<\infty .
$$

Soit $\chi$ la fonction caractéristique des $p$ tels que $|h(p)-1| \geq 1 / 2$. On a

$$
\chi(p)=1 \Rightarrow \frac{1}{4} \leq|h(p)-1|^{2} \leq 2 \operatorname{Re}(1-h(p)),
$$

ce qui donne

$$
\forall p, \quad \chi(p)|h(p)| \leq 8(1-\operatorname{Re} h(p)) .
$$

Or $\sum_{p}(1-\operatorname{Re} h(p)) / p<\infty$, donc

$$
\sum_{\substack{p \\|h(p)-1| \geq 1 / 2}} \frac{|h(p)|}{p}=\sum_{p} \frac{\chi(p)|h(p)|}{p}<\infty .
$$

Enfin

$$
\sum_{k \geq 2} \frac{\left|h\left(p^{k}\right)\right|}{p^{k}} \leq \sum_{k \geq 2} \frac{1}{p^{k}}=\frac{1}{p(p-1)}
$$

d'où

$$
\sum_{p} \sum_{k \geq 2} \frac{\left|h\left(p^{k}\right)\right|}{p^{k}}<\infty .
$$

(H1) est donc vraie, ce qui termine la preuve du corollaire.

\section{Démonstration du théorème 2}

(a) Le cas $1 \leq y<2$. Le résultat se déduit du théorème $\mathrm{E}$ par convolution. Soit $g$ une fonction vérifiant les hypothèses du théorème 2 . On peut supposer

$$
\sum_{p} \frac{y-|g(p)|}{p}<\infty
$$


car sinon on a directement, pour tout $\theta$,

$$
|G(x, \theta)| \leq \sum_{n \leq x}|g(n)|=o\left(x(\log x)^{y-1}\right) .
$$

Définissons une fonction $f$ complètement multiplicative de la manière suivante :

- Si $g(p)=r_{p} e^{i \theta_{p}}$, avec $0<r_{p} \leq y$, on pose $f(p)=y e^{i \theta_{p}}$.

- Si $g(p)=0$, on pose $f(p)=y e^{i \theta_{p}}$, avec $\theta_{p}$ quelconque.

On peut écrire $g=f * h$, les séries entières correspondantes vérifiant

$$
\forall z,|z|<1, \quad \sum_{\nu=0}^{\infty} g\left(p^{\nu}\right) z^{\nu}=\left(\sum_{\nu=0}^{\infty} f\left(p^{\nu}\right) z^{\nu}\right)\left(\sum_{\nu=0}^{\infty} h\left(p^{\nu}\right) z^{\nu}\right),
$$

d'où, puisque $f$ est complètement multiplicative,

$$
\sum_{\nu=0}^{\infty} h\left(p^{\nu}\right) z^{\nu}=(1-f(p) z) \sum_{\nu=0}^{\infty} g\left(p^{\nu}\right) z^{\nu} .
$$

Donc on a $h\left(p^{\nu}\right)=g\left(p^{\nu}\right)-f(p) g\left(p^{\nu-1}\right)$ pour tout $\nu \geq 1$. En particulier, d'après la définition de $f$, on a

$$
|h(p)|=|g(p)-f(p)|=y-|g(p)| .
$$

On en déduit

$$
\begin{aligned}
\sum_{\nu=1}^{\infty} \frac{\left|h\left(p^{\nu}\right)\right|}{p^{\nu}} & =\frac{y-|g(p)|}{p}+\sum_{\nu \geq 2} \frac{\left|g\left(p^{\nu}\right)-f(p) g\left(p^{\nu-1}\right)\right|}{p^{\nu}} \\
& \ll \frac{y-|g(p)|}{p}+\sum_{\nu \geq 2} \frac{\left|g\left(p^{\nu}\right)\right|}{p^{\nu}}+\frac{1}{p^{2}} .
\end{aligned}
$$

Par conséquent, comme on a supposé $\sum_{p}(y-|g(p)|) / p<\infty$, on obtient, en utilisant également l'hypothèse (ii),

$$
\sum_{p} \sum_{\nu \geq 1} \frac{\left|h\left(p^{\nu}\right)\right|}{p^{\nu}}<\infty
$$

puis

$$
\sum_{m=1}^{\infty} \frac{\left|h\left(p^{m}\right)\right|}{p^{m}}<\infty
$$

On peut donc écrire, pour tout $K$,

$$
G(x, \theta)=\sum_{m \leq K} h(m) \sum_{l \leq x / m} f(l) e(\operatorname{lm} \theta)+\sum_{m>K} h(m) \sum_{l \leq x / m} f(l) e(\operatorname{lm} \theta) .
$$


Soit $\varepsilon>0$ fixé. Dans le second terme, la somme intérieure est majorée par $\frac{x}{m}(\log x)^{y-1}$, car $|f|=y^{\Omega}$ avec $0<y<2$, si bien que le second terme est majoré par $\frac{\varepsilon}{2} x(\log x)^{y-1}$, à condition de choisir $K=K(\varepsilon)$ assez grand.

Dans le premier terme, pour chaque $m \leq K$, on peut appliquer le théorème $\mathrm{E}$ à la somme intérieure, ce qui donne à nouveau - en choisissant $x \geq x_{0}(\varepsilon)$ - un terme majoré par $\frac{\varepsilon}{2} x(\log x)^{y-1}$.

Le théorème 2 est ainsi démontré dans le cas où $1 \leq y<2$.

(b) Le cas $0<y<1$. La démonstration précédente se transpose à ce cas presque sans changement. Dans le second terme de $G(x, \theta)$, la somme intérieure est majorée par $\frac{x}{m}\left(\log \frac{2 x}{m}\right)^{y-1}$ et la condition (ii) du théorème assure que pour $K=K(\varepsilon)$ assez grand et $x \geq x_{1}(\varepsilon)$,

$$
\sum_{K<m \leq x} \frac{|h(m)|}{m}\left(\log \frac{2 x}{m}\right)^{y-1} \leq \frac{\varepsilon}{2} x(\log x)^{y-1} .
$$

(c) Le cas général. En utilisant la même méthode que dans la démonstration du théorème 1 , on va montrer que si le théorème 2 est vrai pour $y-1$, alors il est également vrai pour $y$. Ceci, joint à ce qui précède, montrera que le théorème 2 est vrai pour tout $y>0$.

Soit donc $g$ une fonction multiplicative vérifiant les hypothèses du théorème 2 pour un certain $y>1$. Définissons alors les fonctions multiplicatives $f$ et $h$ de la façon suivante :

Si $g(p)=y u_{p}$, avec $\left|u_{p}\right| \leq 1$, on pose

$$
\forall k \geq 0, \quad f\left(p^{k}\right)=d_{y-1}\left(p^{k}\right) u_{p}^{k}, \quad h\left(p^{k}\right)=u_{p}^{k} .
$$

On peut alors écrire $g=f * h * \psi$, avec, en ce qui concerne les séries entières,

$$
\forall z,|z|<1, \quad \sum_{\nu=0}^{\infty} g\left(p^{\nu}\right) z^{\nu}=\frac{1}{\left(1-u_{p} z\right)^{y-1}} \cdot \frac{1}{1-u_{p} z} \sum_{\nu=0}^{\infty} \psi\left(p^{\nu}\right) z^{\nu} .
$$

Donc

$$
\sum_{\nu=0}^{\infty} \psi\left(p^{\nu}\right) z^{\nu}=\left(1-u_{p} z\right)^{y} \sum_{\nu=0}^{\infty} g\left(p^{\nu}\right) z^{\nu}
$$

On obtient ainsi

$$
\forall k \geq 0, \quad \psi\left(p^{k}\right)=\sum_{\nu=0}^{k}\left(-u_{p}\right)^{\nu}\left(\begin{array}{l}
y \\
\nu
\end{array}\right) g\left(p^{k-\nu}\right),
$$

avec, comme $y>0$,

$$
\left|\left(\begin{array}{l}
y \\
\nu
\end{array}\right)\right|=\left|\frac{y(y-1) \ldots(y-\nu+1)}{\nu !}\right| \leq \frac{y(y+1) \ldots(y+\nu-1)}{\nu !}=d_{y}\left(p^{\nu}\right) .
$$


En tenant compte du fait que $\psi(p)=0$, on trouve

$$
\begin{aligned}
\sum_{k=1}^{\infty} \frac{\left|\psi\left(p^{k}\right)\right|}{p^{k}} & \leq \sum_{k=2}^{\infty} \frac{1}{p^{k}} \sum_{\nu=0}^{k} d_{y}\left(p^{\nu}\right)\left|g\left(p^{k-\nu}\right)\right| \\
& \leq \sum_{k=2}^{\infty} \frac{d_{y}\left(p^{k-1}\right) y+d_{y}\left(p^{k}\right)}{p^{k}}+\sum_{\nu=2}^{\infty} \frac{\left|g\left(p^{\nu}\right)\right|}{p^{\nu}} \sum_{k \geq 0} \frac{d_{y}\left(p^{k}\right)}{p^{k}}
\end{aligned}
$$

après avoir changé $\nu$ en $k-\nu$ dans le second terme.

Comme

$$
\sum_{k \geq 0} d_{y}\left(p^{k}\right) / p^{k}=(1-1 / p)^{-y}
$$

est borné, on obtient finalement, grâce à l'hypothèse (ii),

$$
\sum_{p} \sum_{k=1}^{\infty} \frac{\left|\psi\left(p^{k}\right)\right|}{p^{k}}<\infty
$$

puis

$$
\sum_{m=1}^{\infty} \frac{|\psi(m)|}{m}<\infty
$$

Il en résulte qu'il suffit de montrer le résultat du théorème pour $f * h$.

Posons donc

$$
S(x, \theta):=\sum_{n \leq x}(f * h)(n) e(n \theta) .
$$

On veut montrer que pour $\theta$ irrationnel, on a

$$
S(x, \theta)=o\left(x(\log x)^{y-1}\right) .
$$

Procédons comme dans la démonstration du théorème 1 . Comme $h$ est de module au plus 1 , on peut prendre $\widetilde{h}=h$ dans le lemme 2 . On écrit ainsi

$$
S(x, \theta)=\sum_{m \leq x} f(m) H\left(\frac{x}{m}, m \theta\right),
$$

où $H$ est défini comme $\widetilde{H}$. Comme on a

$$
\sum_{m \leq x}|f(m)| \leq \sum_{m \leq x} d_{y-1}(m)=o\left(x(\log x)^{y-1}\right)
$$

on peut restreindre la sommation de (14) au domaine $m \leq x / x_{1}(\varepsilon)$ pour toute constante fixée $x_{1}(\varepsilon)$.

Il reste donc à montrer que, pour $z \geq x_{2}(\varepsilon)$,

$$
\sum_{m \leq z / x_{1}(\varepsilon)} f(m) H\left(\frac{z}{m}, m \theta\right) \ll \varepsilon z(\log z)^{y-1} .
$$


En définissant $E(\varepsilon)$ de la même façon que dans la preuve du théorème 1, on obtient de manière analogue que la sous-somme de (15) correspondant à $\{m \theta\} \notin E(\varepsilon)$ est majorée par le membre de droite.

Pour évaluer, pour $z \geq x_{2}(\varepsilon)$, la somme

$$
\sum_{\substack{m \leq z \\\{\theta m\} \in E(\varepsilon)}} \frac{|f(m)|}{m},
$$

on majore à nouveau la fonction indicatrice de $E(\varepsilon)$ par le polynôme trigonométrique

$$
\frac{C}{Q} \sum_{q \leq B} \frac{1}{q} \sum_{\substack{1 \leq a<q \\(a, q)=1}} \sum_{|k| \leq q Q}\left(1-\frac{|k|}{q Q}\right) \cos \left(2 \pi k\left(\theta-\frac{a}{q}\right)\right),
$$

puis on reporte dans (16) et on intervertit les sommations.

Comme $|f|$ vérifie les hypothèses du théorème 2 , que l'on a supposé vrai pour $y-1$, la contribution de chaque $k \neq 0$ est, par sommation d'Abel, $O\left(x(\log x)^{y-1}\right)$. On obtient donc que (16) est $\ll(\varepsilon+o(1))(\log x)^{y-1}$, ce qui termine la démonstration.

6. Démonstration du corollaire 4. Ecrivons $|f|=d_{y} * \psi$. Les séries entières correspondant à ces fonctions multiplicatives vérifient

$$
\forall z,|z|<1, \quad \sum_{\nu=0}^{\infty}\left|f\left(p^{\nu}\right)\right| z^{\nu}=(1-z)^{-y}=\sum_{\nu=0}^{\infty} \psi\left(p^{\nu}\right) z^{\nu} .
$$

On en déduit facilement, pour tout $k$ entier,

$$
\psi\left(p^{k}\right)=\sum_{\nu=0}^{k}(-1)^{\nu}\left(\begin{array}{l}
y \\
\nu
\end{array}\right)\left|f\left(p^{k-\nu}\right)\right|,
$$

d'où

$$
\left|\psi\left(p^{k}\right)\right| \leq \sum_{\nu=0}^{k} d_{y}\left(p^{\nu}\right)\left|f\left(p^{k-\nu}\right)\right| .
$$

En tenant compte de la relation $\psi(p)=|f(p)|-y$, on trouve ainsi

$$
\begin{aligned}
\sum_{k=1}^{\infty} \frac{\left|\psi\left(p^{k}\right)\right|}{p^{k}} & \leq \frac{y-|f(p)|}{p}+\sum_{k \geq 2} \frac{1}{p^{k}} \sum_{\nu=0}^{k} d_{y}\left(p^{\nu}\right)\left|f\left(p^{k-\nu}\right)\right| \\
& \ll \frac{y-|f(p)|}{p}+\sum_{k=2}^{\infty} \frac{d_{y}\left(p^{k}\right)}{p^{k}}+\sum_{\nu=2}^{\infty} \frac{\left|f\left(p^{\nu}\right)\right|}{p^{\nu}}
\end{aligned}
$$


d'où, grâce aux hypothèses (ii) et (iii),

$$
\sum_{p} \sum_{k=1}^{\infty} \frac{\left|\psi\left(p^{k}\right)\right|}{p^{k}}<\infty
$$

et enfin

$$
\sum_{m=1}^{\infty} \frac{|\psi(m)|}{m}<\infty
$$

On en déduit facilement que

$$
\sum_{n \leq x}|f(n)| \sim x(\log x)^{y-1},
$$

d'où le corollaire annoncé.

7. Démonstration du théorème 3. Dans ce qui suit, on aura besoin de l'ordre moyen de la fonction $2^{\Omega}$. Pour la commodité du lecteur, nous rappellerons comment on peut l'obtenir, en procédant par convolution à partir de l'ordre moyen de la fonction $d$.

(a) Identités de convolution. Comme on a affaire à des fonctions multiplicatives, il suffit de vérifier ces identités sur les puissances de nombres premiers.

Lemme 3. On a $2^{\Omega}=h * f$, où $h$ et $f$, multiplicatives, sont définies respectivement par

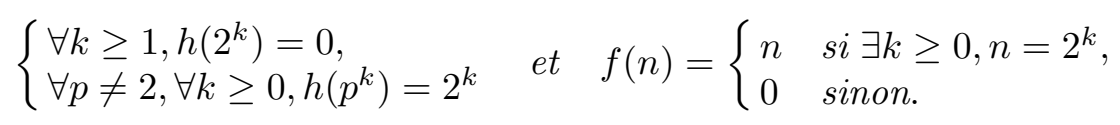

Preuve. Soit $p \neq 2$ et $k \geq 0$. Alors

$$
(h * f)\left(p^{k}\right)=\sum_{\nu=0}^{k} \underbrace{f\left(p^{\nu}\right)}_{=0 \text { si } \nu \neq 0} h\left(p^{k-\nu}\right)=h\left(p^{k}\right)=2^{k}=2^{\Omega\left(p^{k}\right)} .
$$

Prenons $k \geq 0$. On a

$$
(h * f)\left(2^{k}\right)=\sum_{\nu=0}^{k} \underbrace{h\left(2^{\nu}\right)}_{=0 \text { si } \nu>0} f\left(2^{k-\nu}\right)=f\left(2^{k}\right)=2^{k}=2^{\Omega\left(2^{k}\right)} .
$$

Lemme 4. On a $h=d * g$, où $g$, multiplicative, est définie par

$$
\forall p \neq 2, \quad g\left(p^{k}\right)=\left\{\begin{array}{ll}
2^{k-2} & \text { si } k \geq 2, \\
0 & \text { si } k=1
\end{array} \quad \text { et } \quad g\left(2^{k}\right)= \begin{cases}0 & \text { si } k \geq 3, \\
1 & \text { si } k=2, \\
-2 & \text { si } k=1\end{cases}\right.
$$


Preuve. Soit $p \neq 2$ et $k \geq 2$. Alors

$$
\begin{aligned}
(d * g)\left(p^{k}\right) & =\sum_{\nu=0}^{k} g\left(p^{\nu}\right) d\left(p^{k-\nu}\right)=(k+1)+\sum_{\nu=2}^{k} 2^{\nu-2}(k-\nu+1) \\
& =(k+1)+\sum_{\nu=2}^{k} 2^{\nu-2} \sum_{i=0}^{k-\nu} 1=(k+1)+\sum_{i=0}^{k-2} \sum_{\nu=2}^{k-i} 2^{\nu-2} \\
& =(k+1)+\sum_{i=0}^{k-2}\left(2^{k-i-1}-1\right)=1+\sum_{i=0}^{k-1} 2^{i}=2^{k}=h\left(p^{k}\right) .
\end{aligned}
$$

Pour $p \neq 2$ et $k=1$, on a $(d * g)(p)=2=h(p)$.

Pour $p=2$ et $k \geq 2$, on a

$$
(d * g)\left(2^{k}\right)=\sum_{\nu=0}^{k} g\left(2^{\nu}\right) d\left(2^{k-\nu}\right)=(k+1)-2 k+(k-1)=0=h\left(2^{k}\right) .
$$

Pour $p=2$ et $k=1$, on a $(d * g)(2)=0=h(2)$.

(b) Ordres moyens

Lemme 5. Si on pose

$$
C=\prod_{p>2}\left(1+\frac{1}{p(p-2)}\right)
$$

on $a$

$$
\sum_{n \leq x} h(n)=\frac{1}{4} C x \log x+\mathcal{O}(x) .
$$

Preuve. D'après le lemme 4 , on a

$$
\sum_{n \leq x} h(n)=\sum_{k \leq x} g(k) \sum_{l \leq x / k} d(l)=\sum_{k \leq x} g(k)\left(\frac{x}{k} \log \frac{x}{k}+\mathcal{O}\left(\frac{x}{k}\right)\right),
$$

soit

(17) $\sum_{n \leq x} h(n)=x \log x \sum_{k \leq x} \frac{g(k)}{k}-x \sum_{k \leq x} \frac{g(k) \log k}{k}+\mathcal{O}\left(x \sum_{k \leq x} \frac{|g(k)|}{k}\right)$.

Pour tout $\alpha$ réel, on a

$$
\sum_{k \leq x} \frac{|g(k)|}{k^{\alpha}} \leq \prod_{p \leq x}\left(\sum_{\nu \geq 0} \frac{\left|g\left(p^{\nu}\right)\right|}{p^{\alpha \nu}}\right) .
$$

La série $\sum_{\nu}\left|g\left(p^{\nu}\right)\right| / p^{\alpha \nu}$ converge dès que $\alpha$ est tel que $3^{\alpha}>2$ (pour $p=2$, il n'y a qu'un nombre fini de termes non nuls dans la série). Pour 
$\alpha>(\log 2) / \log 3$, on obtient ainsi

$$
\sum_{k \leq x} \frac{|g(k)|}{k^{\alpha}} \ll \prod_{2<p \leq x}\left(1+\sum_{\nu \geq 2} \frac{2^{\nu-2}}{p^{\alpha \nu}}\right)=\prod_{2<p \leq x}\left(1+\frac{1}{p^{\alpha}\left(p^{\alpha}-2\right)}\right),
$$

ce qui montre que la série $\sum g(k) / k^{\alpha}$ converge absolument $(\alpha>(\log 2) / \log 3$ $\Rightarrow 2 \alpha>1)$.

On peut donc écrire

$$
\sum_{k \leq x} \frac{g(k)}{k}=\sum_{k=1}^{\infty} \frac{g(k)}{k}-\sum_{k>x} \frac{g(k)}{k}
$$

avec

$$
\text { (18) } \sum_{k=1}^{\infty} \frac{g(k)}{k}=\prod_{p}\left(\sum_{\nu \geq 0} \frac{g\left(p^{\nu}\right)}{p^{\nu}}\right)=\left(1-\frac{2}{2}+\frac{1}{4}\right) \prod_{p>2}\left(1+\frac{1}{p(p-2)}\right)=\frac{C}{4}
$$

et, en s'inspirant de la méthode de Rankin, et en prenant $\alpha>(\log 2) / \log 3$,

$$
\sum_{k>x} \frac{g(k)}{k} \ll \frac{1}{x^{1-\alpha}} \sum_{k>x} \frac{|g(k)|}{k^{\alpha}} \ll_{\alpha} \frac{1}{x^{1-\alpha}} .
$$

Par ailleurs, si $(\log 2) / \log 3<\alpha<1$, on a

$$
\sum_{k \leq x} \frac{g(k) \log k}{k} \ll_{\alpha} \sum_{k \leq x} \frac{|g(k)|}{k^{\alpha}} \ll_{\alpha} 1 .
$$

En choisissant $\alpha$ quelconque dans ] $(\log 2) / \log 3,1[$ et en reportant (18)-(20) dans (17), on obtient le résultat annoncé.

Lemme 6. Avec la même constante $C$ que dans le lemme 5 , on a

$$
\sum_{n \leq x} 2^{\Omega(n)}=\frac{C}{8 \log 2} x(\log x)^{2}+\mathcal{O}(x \log x) .
$$

Preuve. En utilisant le lemme 3, on peut écrire

$$
\sum_{n \leq x} 2^{\Omega(n)}=\sum_{k \leq x} f(k) \sum_{l \leq x / k} h(l),
$$

ce qui donne, d'après le lemme 5 ,

$$
\sum_{n \leq x} 2^{\Omega(n)}=\sum_{k \leq x} f(k)\left(\frac{1}{4} C \frac{x}{k} \log \frac{x}{k}+\mathcal{O}\left(\frac{x}{k}\right)\right)
$$

soit

$$
\sum_{n \leq x} 2^{\Omega(n)}=\frac{C}{4} x \log x \sum_{k \leq x} \frac{f(k)}{k}-\frac{C}{4} x \sum_{k \leq x} \frac{f(k) \log k}{k}+\mathcal{O}\left(x \sum_{k \leq x} \frac{f(k)}{k}\right) .
$$


Or on a

$$
\sum_{k \leq x} \frac{f(k)}{k}=\sum_{0 \leq r \leq \log _{2} x} 1=\frac{\log x}{\log 2}+\mathcal{O}(1)
$$

et

$$
\begin{aligned}
\sum_{k \leq x} \frac{f(k) \log k}{k} & =\sum_{0 \leq r \leq \log _{2} x} \log 2^{r}=\log 2 \frac{\left\lfloor\log _{2} x\right\rfloor\left(\left\lfloor\log _{2} x\right\rfloor+1\right)}{2} \\
& =\frac{\log 2}{2}\left(\frac{\log x}{\log 2}+\mathcal{O}(1)\right)^{2}=\frac{(\log x)^{2}}{2 \log 2}+\mathcal{O}(\log x)
\end{aligned}
$$

On trouve donc finalement

$$
\sum_{n \leq x} 2^{\Omega(n)}=\frac{C}{8 \log 2} x(\log x)^{2}+\mathcal{O}(x \log x) .
$$

(c) Minoration de la somme d'exponentielles. On notera $S(x, \theta)=$ $\sum_{n \leq x} 2^{\Omega(n)} e(n \theta)$. Le principe de l'hyperbole, avec le lemme 3 , donne

$$
S(x, \theta)=\sum_{l \leq x} f(l) \sum_{k \leq x / l} h(k) e(k l \theta)=S_{1}(\theta)+S_{2}(\theta)
$$

avec

$$
S_{1}(\theta)=\sum_{l \leq y} f(l) \sum_{k \leq x / l} h(k) e(k l \theta)
$$

et

$$
S_{2}(\theta)=\sum_{k \leq x / y} h(k) \sum_{y<l \leq x / k} f(l) e(l k \theta) .
$$

On a tout d'abord

$$
\left|S_{1}(\theta)\right| \leq \sum_{l \leq y} f(l) \sum_{k \leq x / l} h(k),
$$

ce qui donne, d'après le lemme 5 ,

$$
S_{1}(\theta) \ll \sum_{l \leq y} f(l) \frac{x}{l} \log x=x \log x \sum_{2^{r} \leq y} 1,
$$

d'où finalement

$$
S_{1}(\theta) \ll x \log x \log y .
$$

La deuxième somme peut s'écrire sous la forme $S_{2}(\theta)=T_{1}(\theta)+T_{2}(\theta)$ avec

$$
\begin{gathered}
T_{1}(\theta)=\sum_{x /(4 y)<k \leq x / y} h(k) \sum_{y<l \leq x / k} f(l) e(l k \theta), \\
T_{2}(\theta)=\sum_{k \leq x /(4 y)} h(k) \sum_{2^{r} \leq x / k} 2^{r} e\left(2^{r} k \theta\right) .
\end{gathered}
$$


Dans la somme $T_{1}(\theta)$, comme $x / k<4 y$, il y a au plus deux puissances de 2 dans $] y, x / k]$, d'où

$$
\left|T_{1}(\theta)\right| \leq \sum_{x /(4 y)<k \leq x / y} h(k)\left(\frac{x}{k}+\frac{x}{2 k}\right) \leq 6 y \sum_{k \leq x / y} h(k),
$$

ce qui, grâce au lemme 5 , donne

$$
T_{1}(\theta) \ll x \log x .
$$

Dans $T_{2}(\theta)$, pour chaque $k$, ce sont les deux plus grandes valeurs de $r$ qui jouent un rôle prépondérant. On écrit donc $T_{2}(\theta)=U_{1}(\theta)+U_{2}(\theta)$, avec

$$
\begin{gathered}
U_{1}(\theta)=\sum_{k \leq x /(4 y)} h(k) \sum_{\log _{2} y<r<\left\lfloor\log _{2}(x / k)\right\rfloor-1} 2^{r} e\left(2^{r} k \theta\right), \\
U_{2}(\theta)=\sum_{k \leq x /(4 y)} h(k) 2^{\left\lfloor\log _{2}(x / k)\right\rfloor-1} e\left(2^{\left\lfloor\log _{2}(x / k)\right\rfloor-1} k \theta\right)\left(1+2 e\left(2^{\left\lfloor\log _{2}(x / k)\right\rfloor-1} k \theta\right)\right) .
\end{gathered}
$$

La condition $k \leq x /(4 y)$ assure que l'on a $\left\lfloor\log _{2}(x / k)\right\rfloor-1>\log _{2} y$.

Une majoration grossière donne

$$
\begin{aligned}
\left|U_{1}(\theta)\right| & \leq \sum_{k \leq x /(4 y)} h(k) \sum_{\log _{2} y<r<\left\lfloor\log _{2}(x / k)\right\rfloor-1} 2^{r} \\
& \leq \sum_{k \leq x /(4 y)} h(k)\left(2^{\left\lfloor\log _{2}(x / k)\right\rfloor-1}-1\right) \leq \frac{x}{2} \sum_{k \leq x} \frac{h(k)}{k} .
\end{aligned}
$$

Or, en effectuant une sommation par parties, on obtient, avec le lemme 5,

$$
\sum_{k \leq x} \frac{h(k)}{k}=\frac{1}{x} \sum_{k \leq x} h(k)+\int_{1}^{x} \frac{d u}{u^{2}} \sum_{k \leq u} h(k)=\frac{C}{4} \int_{1}^{x} \frac{\log u d u}{u}+\mathcal{O}(\log x),
$$

soit

$$
\sum_{k \leq x} \frac{h(k)}{k}=\frac{C}{8}(\log x)^{2}+\mathcal{O}(\log x)
$$

D'où finalement,

$$
\left|U_{1}(\theta)\right| \leq \frac{C}{16} x(\log x)^{2}+\mathcal{O}(x \log x) .
$$

C'est la somme $U_{2}(\theta)$ que l'on va chercher à minorer:

$\left|U_{2}(\theta)\right| \geq \operatorname{Re} U_{2}(\theta)$

$=\sum_{k \leq x /(4 y)} h(k) 2^{\left\lfloor\log _{2}(x / k)\right\rfloor-1}\left(\cos \left(2^{\left\lfloor\log _{2}(x / k)\right\rfloor} k \pi \theta\right)+2 \cos \left(2^{\left\lfloor\log _{2}(x / k)\right\rfloor+1} k \pi \theta\right)\right)$. 
Or pour tout $\alpha$, on a l'inégalité $\cos (2 \pi \alpha) \geq 1-2 \pi\|\alpha\|$, donc $\left|U_{2}(\theta)\right|$

$\geq \sum_{k \leq x /(4 y)} h(k) 2^{\left\lfloor\log _{2}(x / k)\right\rfloor-1}\left(3-2 \pi\left\|2^{\left\lfloor\log _{2}(x / k)\right\rfloor-1} k \theta\right\|-4 \pi\left\|2^{\left\lfloor\log _{2}(x / k)\right\rfloor} k \theta\right\|\right)$.

On a l'encadrement suivant :

$$
x / 2 \leq 2^{\left\lfloor\log _{2}(x / k)\right\rfloor} k \leq x,
$$

ce qui donne

$$
\left|U_{2}(\theta)\right| \geq(1-2 \pi M(x, y, \theta)) U_{2}(0)
$$

si on pose

$$
M(x, y, \theta)=\max _{\substack{x / 4 \leq n \leq x \\ 2^{\left\lfloor\log _{2} y\right\rfloor} \mid n}}\|n \theta\| .
$$

Or d'après les majorations (21) et (22), on a

$$
U_{2}(\theta)=S(x, \theta)-U_{1}(\theta)+\mathcal{O}(x \log x \log y) .
$$

Par conséquent, en reportant dans (24), on obtient

$|S(x, \theta)|+\left|U_{1}(\theta)\right| \geq(1-2 \pi M(x, y, \theta))\left(S(x, 0)-U_{1}(0)\right)+\mathcal{O}(x \log x \log y)$,

soit, d'après le lemme 6 et la majoration (23),

$|S(x, \theta)| \geq \frac{C}{16}\left(\left(\frac{2}{\log 2}-1\right)(1-2 \pi M(x, y, \theta))-1\right) x(\log x)^{2}+\mathcal{O}(x \log x \log y)$.

On obtient finalement, uniformément en $x, y$ et $\theta$,

(25) $|S(x, \theta)| \geq((1-\log 2)-\pi(2-\log 2) M(x, y, \theta)) S(x, 0)+\mathcal{O}(x \log x \log y)$.

(d) Étude de $M(x, y, \theta)$. On va montrer le résultat suivant:

Lemme 7. Soit $\phi: \mathbb{R}_{+}^{*} \rightarrow \mathbb{R}$ telle que $\lim _{x \rightarrow \infty} \phi(x)=\infty$. Soit $\varepsilon>0$. Alors il existe un irrationnel $\theta_{\phi, \varepsilon}$ et une suite strictement croissante $\left(x_{q}\right)$ d'entiers naturels telle que

$$
\forall q \in \mathbb{N}, \quad M\left(x_{q}, \phi\left(x_{q}\right), \theta_{\phi, \varepsilon}\right) \leq \varepsilon .
$$

Preuve. On cherche $\theta_{\phi, \varepsilon}$ sous la forme

$$
\theta_{\phi, \varepsilon}=\sum_{\nu=0}^{\infty} 2^{-\alpha_{\nu}},
$$


où $\left(\alpha_{\nu}\right)$ est une suite strictement croissante d'entiers naturels. Pour tout $n \in[x / 4, x]$ tel que $n=2^{\left\lfloor\log _{2} y\right\rfloor} k(k \in \mathbb{N})$, on a

$$
\begin{aligned}
\left\|n \theta_{\phi, \varepsilon}\right\| & =\left\|k \sum_{\nu=0}^{\infty} 2^{\left\lfloor\log _{2} y\right\rfloor-\alpha_{\nu}}\right\|=\left\|k \sum_{\alpha_{\nu}>\left\lfloor\log _{2} y\right\rfloor} 2^{\left\lfloor\log _{2} y\right\rfloor-\alpha_{\nu}}\right\| \\
& \leq k 2^{\left\lfloor\log _{2} y\right\rfloor+1-\min \left\{\alpha_{\nu}>\left\lfloor\log _{2} y\right\rfloor\right\}}
\end{aligned}
$$

soit

$$
M\left(x, y, \theta_{\phi, \varepsilon}\right) \leq \frac{2 x}{2^{\min \left\{\alpha_{\nu}>\left\lfloor\log _{2} y\right\rfloor\right\}}} .
$$

On définit alors la suite $\left(x_{q}\right)_{q \in \mathbb{N}}$ de la manière suivante. On pose

$$
\left\{\begin{array}{l}
x_{0} \in \mathbb{N}^{*} \text { quelconque, } \\
\alpha_{0}=\max \left\{1+\left\lfloor\log _{2} \phi\left(x_{0}\right)\right\rfloor, 2+\left\lfloor\log _{2} x_{0}-\log _{2} \varepsilon\right\rfloor\right\}
\end{array}\right.
$$

puis, par récurrence

$$
\left\{\begin{array}{l}
x_{q+1}>x_{q} \text { tel que }\left\lfloor\log _{2} \phi\left(x_{q+1}\right)\right\rfloor \geq \alpha_{q}, \\
\alpha_{q+1}=\max \left\{1+\left\lfloor\log _{2} \phi\left(x_{q+1}\right)\right\rfloor, 2+\left\lfloor\log _{2} x_{q+1}-\log _{2} \varepsilon\right\rfloor, \alpha_{q}+(q+1)\right\} .
\end{array}\right.
$$

Comme on a

$$
\forall q \in \mathbb{N}, \quad \alpha_{q+1} \geq \alpha_{q}+(q+1),
$$

la suite $\left(\alpha_{q}\right)$ est strictement croissante. De plus $\theta_{\phi, \varepsilon}$ est irrationnel (sinon $\left(\alpha_{q}\right)$ serait périodique et $\alpha_{q+1}-\alpha_{q}$ serait borné).

Soit $q \in \mathbb{N}$. Par construction, on a

$$
\alpha_{q}=\min \left\{\alpha_{\nu}>\left\lfloor\log _{2} \phi\left(x_{q}\right)\right\rfloor\right\} .
$$

En effet, $\alpha_{q}>\left\lfloor\log _{2} \phi\left(x_{q}\right)\right\rfloor$ et $\forall \nu<q, \alpha_{\nu} \leq \alpha_{q-1} \leq\left\lfloor\log _{2} \phi\left(x_{q}\right)\right\rfloor$. Donc, d'après (26), on a bien défini une suite strictement croissante $\left(x_{q}\right)$ telle que

$$
\forall q \in \mathbb{N}, \quad M\left(x_{q}, \phi\left(x_{q}\right), \theta_{\phi, \varepsilon}\right) \leq 2 x_{q} / 2^{\alpha_{q}} \leq \varepsilon .
$$

(e) Conclusion. On peut maintenant regrouper tous les résultats obtenus précédemment. On choisit $\varepsilon>0$ tel que

$$
(1-\log 2)-\pi(2-\log 2) \varepsilon=A>0 .
$$

On prend la fonction $\phi=\log$. Elle vérifie bien les hypothèses du lemme 7, ce qui fournit un irrationnel $\theta_{\log , \varepsilon}$ et une suite $\left(x_{q}\right)$. On a alors, d'après $(25)$,

$$
\forall q \in \mathbb{N}, \quad\left|S\left(x_{q}, \theta_{\log , \varepsilon}\right)\right| \geq A S\left(x_{q}, 0\right)+\mathcal{O}\left(x_{q} \log x_{q} \log \log x_{q}\right),
$$

où $\left(x_{q}\right)$ est une suite strictement croissante d'entiers naturels. Donc

$$
\left|S\left(x, \theta_{\log , \varepsilon}\right)\right|=\Omega(S(x, 0)),
$$

ce qui termine la démonstration du théorème 3. 


\section{Références}

[1] S. Chowla, Some problems of diophantine approximation (I), Math. Z. 33 (1931), $544-563$.

[2] H. Daboussi, On some exponential sums, in: Analytic Number Theory, Proceedings of a Conference in honour of Paul T. Bateman, Birkhäuser, Boston, 1990, $111-118$.

[3] H. Daboussi et H. Delange, Quelques propriétés des fonctions multiplicatives de module au plus égal à 1, C. R. Acad. Sci. Paris Sér. A 278 (1974), 657-660.

[4] - - -, On multiplicative arithmetical functions whose modulus does not exceed one, J. London Math. Soc. (2) 26 (1982), 245-264.

[5] H. Delange, Sur les fonctions arithmétiques multiplicatives, Ann. Sci. École Norm. Sup. (3) 78 (1961), 273-304.

[6] Y. Dupain, R. R. Hall et G. Tenenbaum, Sur l'équirépartition modulo 1 de certaines fonctions de diviseurs, J. London Math. Soc. (2) 26 (1982), 397-411.

[7] P. Erdős, Some remarks on diophantine approximations, J. Indian Math. Soc. 12 (1948), 67-74.

[8] K. H. Indlekofer, Properties of uniformly summable multiplicative functions, Period. Math. Hungar. 17 (1986), 143-161.

[9] H. L. Montgomery and R. C. Vaughan, On exponential sums with multiplicative coefficients, Invent. Math. 43 (1977), 69-82.

[11] G. Tenenbaum, Introduction à la théorie analytique et probabiliste des nombres, Publ. Inst. Élie Cartan, Nancy, 1990.

URA D0752

DÉPARTEMENT DE MATHÉMATIQUES

BÂTIMENT 425

UNIVERSITÉ DE PARIS XI, ORSAY

91405 ORSAY CEDEX, FRANCE 